cohort. We estimated the annual age- and sex- standardized proportion of patients who filled prescriptions for at least one of the drugs of interest (table).

Results A total of 30787 patients with prevalent SLE were identified, of whom 5267 had LN. Mean age (SD) was 49.3 (14.1) years for SLE and 51.9 (17.2) years for LN. Ninety percent were female, $61.0 \%$ White, $17.4 \%$ Black, $11.2 \%$ Hispanic, 3.0\% Asian and 7.0\% other/unknown. Use and time trends of therapeutic agents for 2006-2016 are included in the table. Anti-malarials were the most frequently used drug class, with some temporal change over the course of the study; $50.1 \%$ in 2006 to $47.5 \%$ in 2016 for SLE, and $38.8 \%$ in 2006 to $45.1 \% 2016$ for LN. Prednisone was the second most frequently used drug, decreasing from $40.0 \%$ in 2006 to $34.7 \%$ in 2016; use of methylprednisolone and other systemic glucocorticoids increased during that time period. Methotrexate was the most commonly used nonbiologic immunosuppressive drug in SLE (8.0\%), while MMF was most frequently prescribed in LN (15.0\%). Cyclophosphamide use declined over the last decade for SLE $(1.2 \%$ to $0.3 \%$ in 2016) and $\mathrm{LN}(4.2 \%$ to $1.3 \%$ in 2016). Belimumab was the most commonly used biologic for SLE with a slight uptrend since approval. Rituximab use increased in SLE and was the most commonly used biologic for LN. At any point in time $\sim 24 \%$ of the patients were not receiving any of the medications of interest.

Conclusions Over the past decade, a substantial proportion of patients with SLE and LN continued to receive glucocorticoids. The proportion of patients receiving nonbiologic immunosuppressants remained stable, however there was a progressive decline in the use of cyclophosphamide. Since 2011 the proportion of patients receiving rituximab is higher than for cyclophosphamide. The increasing use of rituximab, which is not currently approved for SLE or LN, highlights the need to clarify its therapeutic role in these diseases.

Funding Source(s): None

\section{IMPACT OF THE HOP-STEP PROGRAM IN IMPROVING PROVIDER KNOWLEDGE AND SKILLS FOR LUPUS PREGNANCY PLANNING AND MANAGEMENT}

Ravyn S Njagu*, Amanda M Eudy, Amanda Snyderman, Lisa G Criscione-Schreiber, Megan EB Clowse. Duke University

\subsection{6/lupus-2019-|sm.113}

Background The HOP-STEP Program (Healthy Outcomes in Pregnancy with SLE through Education of Providers) is designed to optimize lupus pregnancy outcomes through providing physicians with the necessary knowledge, skills and resources to guide pregnancy planning and management. The goal of this analysis was to measure the impact of the HOPSTEP program.

Methods The in-person component of HOP-STEP was presented at the Association of Women in Rheumatology (AWIR) Conference in August 2018. The workshop included a presentation, peer roll-playing workshop, patient simulation, and distribution of Preparing for Pregnancy Checklist and Birth Control for Women with Lupus handouts. A survey was emailed to 149 individuals before and after workshop completion.

Results The analysis included 68 pre-surveys (response rate 46\%, 93\% women, 66\% attending-level rheumatologists) and
55 post-surveys (response rate 37\%, 95\% women, 62\% attending-level rheumatologists).

Systematic approach: After the program, more providers had a systematic approach to preparing a woman with lupus for pregnancy $(45.6 \%$ to $94.6 \% ; \mathrm{p}<0.0001)$.

Contraception: There was an increase in correct responses regarding emergency contraception $(47 \%$ to $91 \%, \mathrm{p}<0.0001)$; a decrease in correct responses regarding IUD use $(96 \%$ to $77 \%, \mathrm{p}=0.003)$; and no difference in correct responses to questions regarding thrombotic risk or efficacy of contraception.

Teratogenicity: After the program, fewer providers identified pregnancy-compatible medications (azathioprine and tacrolimus) as teratogenic $(60 \%$ to $9 \% ; \mathrm{p}<0.0001)$. Additionally, more providers identified all three teratogenic medications (methotrexate, mycophenolate, and cyclophosphamide) $(80.9 \%$ to $92.7 \% ; \mathrm{p}=0.07)$.

Birth Defects: Participants were asked to put medications in order from most to least likely to cause birth defects, the correct order being mycophenolate or cyclophosphamide, methotrexate, then leflunomide. No difference was observed in correctly ordered medications $(16.2 \%$ to $16.4 \%$; $p=1.0)$. However, there was an increase in providers who correctly identified mycophenolate as the most or second-most teratogenic $(13 \%$ to $44 \% ; \mathrm{p}=0.02)$.

Provider Confidence: Participants were asked to rank their confidence on a scale of 0 (not confident) to 100 (very confident). Median provider confidence increased for helping women with lupus choose appropriate contraception (59 to $89 ; \mathrm{p}<0.0001)$ and medications compatible with pregnancy (66 to $91 ; \mathrm{p}<0.0001$ ).

Conclusions After participation in the in-person HOP-STEP program, providers had increased knowledge and confidence in pregnancy planning and management in lupus patients. The program emphasized skills over knowledge, as is reflected in the survey results. The handouts used in the program provide all of the necessary information, which can overcome existing and ongoing gaps in rheumatologists knowledge.

Funding Source(s): GlaxoSmithKline

\section{FEATURES OF FIBROMYALGIA IN LUPUS NEPHRITIS}

Jennifer L Rogers*, Amanda M Eudy, Lisa G Criscione-Schreiber, David S Pisetsky, Jayanth Doss, Kai Sun, Megan EB Clowse. Duke University

\subsection{6/lupus-2019-Ism.114}

Background Patients with SLE have poor health related quality of life (HRQoL), however the predominant causes for decreased HRQoL in different subgroups of SLE are not well understood. Features of fibromyalgia, including fatigue, widespread pain, depression, sleep and cognitive dysfunction, are prevalent in SLE affecting 20\%-65\% of patients and often contribute to disability and poor health related quality of life (HRQoL). These aspects of fibromyalgia have not been well described in lupus nephritis (LN). We evaluated self-reported symptoms of fibromyalgia and mood disorders in patients with and without lupus nephritis

Methods This was a cross sectional study of SLE patients (ACR 1997 or SLICC 2012 criteria) from July to November 2018. All patients completed Systemic Lupus Activity Questionnaire (SLAQ), Patient Health Questionnaire-9 (PHQ9), and 